Efficiency of energy conversion for a piezoelectric power harvesting system

This article has been downloaded from IOPscience. Please scroll down to see the full text article.

2006 J. Micromech. Microeng. 162429

(http://iopscience.iop.org/0960-1317/16/11/026)

The Table of Contents and more related content is available

Download details:

IP Address: 140.112.113.225

The article was downloaded on 22/12/2008 at 01:18

Please note that terms and conditions apply. 


\title{
Efficiency of energy conversion for a piezoelectric power harvesting system
}

\author{
Y C Shu and I C Lien \\ Institute of Applied Mechanics, National Taiwan University, Taipei 106, Taiwan, \\ Republic of China \\ E-mail: yichung@spring.iam.ntu.edu.tw
}

Received 22 June 2006, in final form 7 September 2006

Published 29 September 2006

Online at stacks.iop.org/JMM/16/2429

\begin{abstract}
This paper studies the energy conversion efficiency for a rectified piezoelectric power harvester. An analytical model is proposed, and an expression of efficiency is derived under steady-state operation. In addition, the relationship among the conversion efficiency, electrically induced damping and ac-dc power output is established explicitly. It is shown that the optimization criteria are different depending on the relative strength of the coupling. For the weak electromechanical coupling system, the optimal power transfer is attained when the efficiency and induced damping achieve their maximum values. This result is consistent with that observed in the recent literature. However, a new finding shows that they are not simultaneously maximized in the strongly coupled electromechanical system.
\end{abstract}

\section{Introduction}

Advances in low-power electronic design and fabrication have opened the possibility of self-powered microsensors and communication nodes [1]. At the same time, the need to power remote systems or embedded devices independently has motivated many research efforts harvesting electric energy from various ambient sources, including solar power, thermal gradients and vibration [2]. Among these energy scavenging sources, mechanical vibration is a potential power source that is abundant enough to be of use, is easily accessible through microelectromechanical systems (MEMS) technology for conversion to electric energy, and is ubiquitous in applications ranging from small household appliances to large infrastructures [3, 4].

Piezoelectric vibration-to-electricity converters have received much attention as transducers, since they have high electromechanical coupling, require no external voltage source and are particularly attractive for use in MEMS [5-8]. As a result, piezoelectric materials for scavenging energy from ambient vibration sources have recently seen a dramatic rise in use for power harvesting. This includes the use of resonant piezoelectric-based structures of cantilever beam configuration [9-15] as well as plate (membrane) configuration [16-19]. Other harvesting schemes include the use of long strips of piezoelectric polymers in ocean or river-water flows [20, 21], the use of piezoelectric 'cymbal' transducers [22, 23], and the use of piezoelectric windmill for generating electric power from wind energy [24].

Jeon et al [5] have successfully developed the first MEMSbased micro-scale power generator using a $\{3-3\}$ mode of PZT transducer. A $170 \mu \mathrm{m} \times 260 \mu \mathrm{m}$ PZT beam has been fabricated, and a maximum dc voltage of $3 \mathrm{~V}$ across the load 10.1 M $\Omega$ has been observed. In addition, the energy density of the power generator has been estimated at around $0.74 \mathrm{mWh} \mathrm{cm}^{-2}$, which compares favourably to the use of lithium ion batteries. Roundy et al [3] subsequently created prototyes of thin PZT structures with a target volume power density of $80 \mu \mathrm{W} \mathrm{cm}{ }^{-3}$. Recently, duToit et al [25] provided in-depth design principles for MEMS-scale piezoelectric energy harvesters and proposed a prototype of $30 \mu \mathrm{W} \mathrm{cm}{ }^{-3}$ from low-level vibration. Related works on the modelling of miniaturized piezoelectric power harvesting devices can be found in [26-28].

As the testing, characterization and fabrication of MEMSscale energy harvesters are not always available compared to the similar tasks in bulk power harvesters, a normalization scheme is particularly useful for comparing the performance of micro-scale power harvesters. One good method uses the parameter of efficiency of mechanical to electric energy conversion. Umeda et al $[29,30]$ have studied the efficiency of mechanical impact energy to electric energy using a 


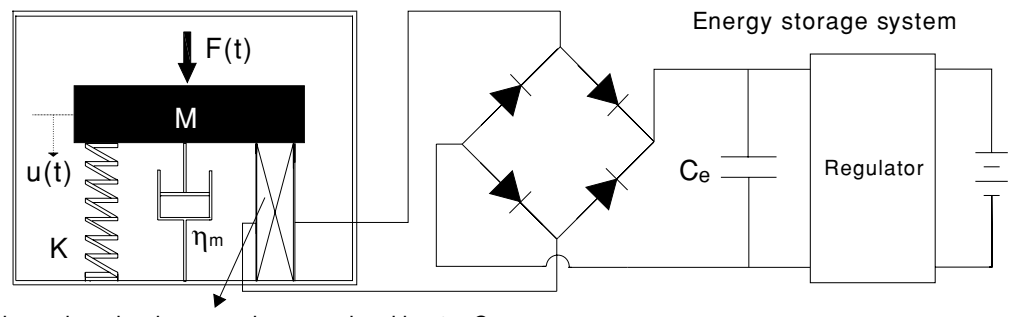

Piezoelectric element characterized by $\Theta \& C_{p}$

Figure 1. An equivalent model for a piezoelectric vibration energy harvesting system.

piezoelectric vibrator. Goldfarb and Jones [31] subsequently investigated the efficiency of the piezoelectric material in a stack configuration for converting mechanical harmonic excitation into electric energy. Roundy [32] provided an expression for effectiveness that can be used to compare various approaches and designs for vibration-based energy harvesting devices. Recently, in contrast to efforts where the conversion efficiency was examined numerically [29], Richards et al [33] derived an analytic formula to predict the energy conversion efficiency of piezoelectric energy harvesters in the case of ac power output. Since the electronic load requires a stabilized dc voltage while a vibrating piezoelectric element generates an ac voltage, the desired output needs to be rectified, filtered and regulated to ensure the electric compatibility. Thus, we investigate the conversion efficiency for a rectified piezoelectric power harvesting system based on the analysis proposed by Shu and Lien [34] in section 2. We show that the conversion efficiency is dependent on the frequency ratio, the normalized resistance and, in particular, the ratio of the electromechanical coupling coefficient to the mechanical damping ratio. In general, the conversion efficiency can be improved with a larger coupling coefficient and smaller damping. Recently, Cho et al $[35,36]$ performed a series of experiments and proposed a set of design guidelines for the performance optimization of micromachined piezoelectric membrane generators by enhancing the electromechanical coupling coefficient.

When an energy harvester is applied to a system, energy is removed from the vibrating structure and supplied to the desired electronic components, resulting in additional damping of the structure $[37,38]$. Because the efficiency is defined as the ratio of the time-averaged power dissipated across the load to that done by the external force, electrically induced damping can be defined explicitly, and its connection to the conversion efficiency is established in section 3 . It is shown that the load to maximize the conversion efficiency is the same as that to maximize the induced damping. However, the extraction of harvested power may not be simultaneously optimized. It is demonstrated in section 4 that optimization criteria vary according to the relative strength of the coupling. The conclusions are drawn in section 5 .

Finally, our result can be applied to the investigation of charging a battery from a vibrating piezoelectric harvester. It is shown that direct charging will result in a low efficiency of energy conversion, since the equivalent impedance of the battery may not match that of the optimal load in most situations. Ottman et al [39, 40] have developed an adaptive electric circuit to optimize the energy transfer from the piezoelectric element to the stored device. It is based on the principle of load impedance adaptation by tuning the load impedance to obtain a higher power flow. Related work based on the synchronous electric charge extraction can be found in [41-44].

\section{A piezoelectric power harvesting model}

Consider an energy conversion device which includes a vibrating piezoelectric structure together with an energy storage system. If the modal density of such a device is widely separated and the structure is vibrating at around its resonance frequency, we may model the power generator as a mass+spring+damper+piezo structure, as schematically shown in figure 1 [33, 39, 44]. It consists of a piezoelectric element coupled to a mechanical structure. In this approach, a forcing function $F(t)$ is applied to the system and an effective mass $M$ is bounded on a spring of effective stiffness $K$, on a damper of coefficient $\eta_{m}$, and on a piezoelectric element characterized by effective piezoelectric coefficient $\Theta$ and capacitance $C_{p}$. These effective coefficients are dependent on the material constants and the design of energy harvesters and can be derived using the standard modal analysis $[25,38,45,46]$.

Let $u$ be the displacement of the mass $M$, and $V_{p}$ be the voltage across the piezoelectric element. The governing equations of the piezoelectric vibrator can be described by $[25,38,43,44]$

$$
\begin{aligned}
M \ddot{u}(t) & +\eta_{m} \dot{u}(t)+K u(t)+\Theta V_{p}(t)=F(t), \\
& -\Theta \dot{u}(t)+C_{p} \dot{V}_{p}(t)=-I(t),
\end{aligned}
$$

where $I(t)$ is the current flowing into the specified circuit. Since most applications of piezoelectric materials for power generation involve the use of periodic straining of piezoelectric elements, the vibrating generator is assumed to be driven at around resonance by the harmonic excitation

$$
F(t)=F_{0} \sin w t
$$

where $F_{0}$ is the constant magnitude and $w$ (in radians per second) is the angular frequency of vibration.

The power generator considered here is connected to a storage circuit system, as illustrated in figure 1. Since the electrochemical battery needs a stabilized dc voltage while a vibrating piezoelectric element generates an ac voltage, this requires a suitable circuit to ensure the electric compatibility. Typically an ac-dc rectifier followed by a filtering capacitance $C_{e}$ is added to smooth the dc voltage, as shown in figure 1 . A controller placed between the rectifier output and the battery is included to regulate the output voltage. Figure 2 is a 


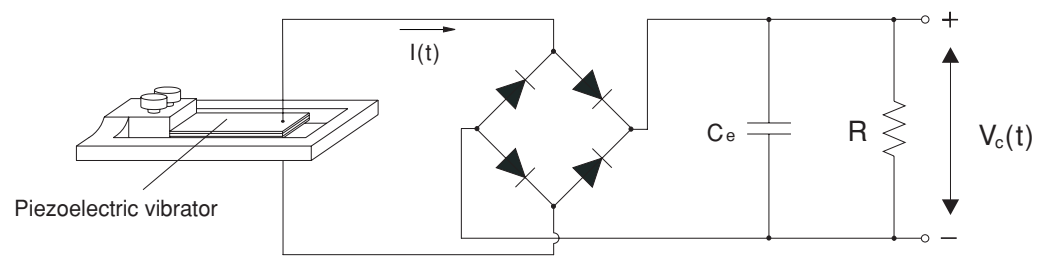

Figure 2. A typical ac-dc harvesting circuit.

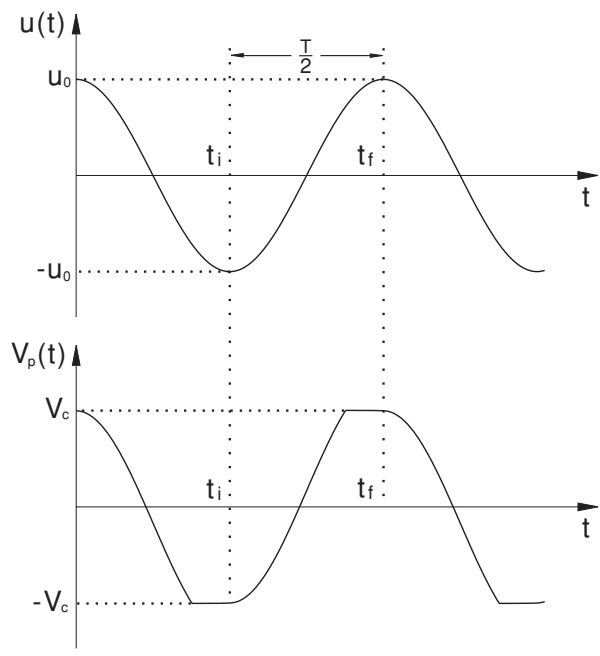

Figure 3. Typical waveforms of displacement $u(t)$ and piezo voltage $V_{p}(t)$ for an ac-dc power harvesting circuit.

simplified energy harvesting circuit commonly adopted for design analysis. It can be used to estimate an upper bound of the real power that the piezoelectric generator is able to deliver at a given excitation. Note that the regulation circuit and battery are replaced with an equivalent resistor $R$ and $V_{c}$ is the rectified voltage across it.

The common approach to having the stable output dc voltage is to assume that the filter capacitor $C_{e}$ is large enough so that the rectified voltage $V_{c}$ is essentially constant [39]. Specifically, $V_{c}(t)=\left\langle V_{c}(t)\right\rangle+V_{\text {ripple }}$, where $\left\langle V_{c}(t)\right\rangle$ and $V_{\text {ripple }}$ are the average and ripple of $V_{c}(t)$, respectively. This average $\left\langle V_{c}(t)\right\rangle$ is independent of $C_{e}$ provided that the time constant $R C_{e}$ is much larger than the oscillating period of the generator [43]. The magnitude of $V_{\text {ripple, }}$, however, depends on $C_{e}$ and is negligible for large $C_{e}$. Under this hypothesis, $V_{c}(t) \approx\left\langle V_{c}(t)\right\rangle$, and therefore in the following, we use $V_{c}$, instead of $\left\langle V_{c}(t)\right\rangle$, to represent the average of $V_{c}(t)$ for notation simplicity.

The rectifying bridge shown in figure 2 is assumed to be perfect here. Thus, it is open circuited if the piezo voltage $\left|V_{p}\right|$ is smaller than the rectified voltage $V_{c}$. As a result, the current flowing into the circuit vanishes, and this implies $\dot{V}_{p}(t)$ varies proportionally with respect to $\dot{u}(t)$, as seen from (2). On the other hand, when $\left|V_{p}\right|$ reaches $V_{c}$, the bridge conducts and the piezo voltage is kept equal to the rectified voltage, i.e., $\left|V_{p}\right|=V_{c}$. Finally, the conduction in the rectifier diodes is blocked again when the absolute value of the piezo voltage $\left|V_{p}(t)\right|$ starts decreasing. Typical waveforms of $u(t)$ and $V_{p}(t)$ satisfying these properties are schematically shown in figure 3 .
To solve (1)-(2) connected to an ac-dc circuit shown in figure 2, we first determine the relation between the average value of the rectified voltage and displacement magnitude. From figure 3, the steady-state solution of $u(t)$ is assumed to take the following form:

$$
u(t)=u_{0} \sin (w t-\theta)
$$

with $u_{0}$ being the constant magnitude. Let $T=\frac{2 \pi}{w}$ be the period of vibration, and $t_{i}$ and $t_{f}$ be two time instants $\left(t_{f}-t_{i}=\frac{T}{2}\right)$ such that the displacement $u$ undergoes from the minimum $-u_{0}$ to the maximum $u_{0}$, as illustrated in figure 3 . Assume that $\dot{V}_{p} \geqslant 0$ during the semi-period from $t_{i}$ to $t_{f}$. It follows that $\int_{t_{i}}^{t_{f}} \dot{V}_{p}(t) \mathrm{d} t=V_{c}-\left(-V_{c}\right)=2 V_{c}$. Note that $C_{e} \dot{V}_{c}(t)+\frac{V_{c}}{R}=0$ for $t_{i}<t<t^{*}$ during which the piezo voltage $\left|V_{p}\right|<V_{c}$ and $I(t)=C_{e} \dot{V}_{c}(t)+\frac{V_{c}}{R}$ for $t^{*} \leqslant t<t_{f}$ during which the rectifier conducts. This gives

$$
-\int_{t_{i}}^{t_{f}} I(t) \mathrm{d} t=-\frac{T}{2} \frac{V_{c}}{R}
$$

since the average current flowing through the capacitance $C_{e}$ is zero, i.e., $\int_{t_{i}}^{t_{f}} C_{e} \dot{V}_{c}(t) \mathrm{d} t=0$ at the steady-state operation. The integration of (2) from time $t_{i}$ to $t_{f}$ is therefore

$$
-2 \Theta u_{0}+2 C_{p} V_{c}=-\frac{T}{2} \frac{V_{c}}{R}
$$

or

$$
V_{c}=\frac{w \Theta R}{w C_{p} R+\frac{\pi}{2}} u_{0} .
$$

Note that (5) is identical with that derived by [39, 43, 44].

We next need to find out $u_{0}$ to determine $V_{c}$. There are two approaches to estimating it in the case of ac-dc power harvesting system in the recent literature [39, 43, 44]. The first one models the piezoelectric device as the current source in parallel with its internal electrode capacitance $C_{p}[5,9$, $11,39]$. It is based on the assumption that the internal current source of the generator is independent of the external load impedance. This is equivalent to assuming that the coupling is very weak and the term $\Theta V_{p}$ can be dropped from (1). On the other hand, if the coupling is not so weak, Guyomar et al [43] and Lefeuvre et al [44] have provided another approach to estimate the displacement magnitude by assuming that the external forcing function and the velocity of the mass are in phase. Recently, Shu and Lien [34] have proposed a new method for determining $u_{0}$ without the uncoupled and in-phase assumptions. They have shown that this new estimation is more accurate than the other two. We here briefly outline the steps of derivation of $u_{0}$ since some of them are required to derive the efficiency of energy conversion.

Consider the balance of energy. Let (1) be multiplied by $\dot{u}(t)$ and (2) be multiplied by $V_{p}(t)$. Integration of the addition 
of these two equations from time $t_{i}$ to $t_{f}$ gives the equation of the energy balance

$\int_{t_{i}}^{t_{f}} F(t) \dot{u}(t) \mathrm{d} t=\int_{t_{i}}^{t_{f}} \eta_{m} \dot{u}^{2}(t) \mathrm{d} t+\int_{t_{i}}^{t_{f}} V_{p}(t) I(t) \mathrm{d} t$,

where

$$
\begin{aligned}
& \int_{t_{i}}^{t_{f}} F(t) \dot{u}(t) \mathrm{d} t=\frac{\pi}{2} F_{0} u_{0} \sin \theta, \\
& \int_{t_{i}}^{t_{f}} \eta_{m} \dot{u}^{2}(t) \mathrm{d} t=\frac{\pi}{2} \eta_{m} w u_{0}^{2}, \\
& \int_{t_{i}}^{t_{f}} V_{p}(t) I(t) \mathrm{d} t=\frac{\pi}{w} \frac{V_{c}^{2}}{R} .
\end{aligned}
$$

Note that (3) and (4) are used to derive (7).

Right now there are two equations (5) and (6) and three unknowns $u_{0}, V_{c}$ and $\theta$. We need the third one to solve them. Differentiating (1) with respect to time $t$ and using (2), we have

$$
\begin{aligned}
M \frac{\mathrm{d}}{\mathrm{d} t} \ddot{u}(t)+\eta_{m} \frac{\mathrm{d}}{\mathrm{d} t} \dot{u}(t) \\
\quad+\left(K+\frac{\Theta^{2}}{C_{p}}\right) \frac{\mathrm{d}}{\mathrm{d} t} u(t)-\frac{\Theta}{C_{p}} I(t)=\frac{\mathrm{d}}{\mathrm{d} t} F(t) .
\end{aligned}
$$

Integrating (8) with respect to time $t$ from $t_{i}$ to $t_{f}$ and using (4) provides the third equation

$$
\left(K-M w^{2}+\frac{\Theta^{2}}{C_{p}}\right) u_{0}-\frac{\pi \Theta}{2 C_{p} w R} V_{c}=F_{0} \cos \theta .
$$

Thus, the unknown variable $\theta$ can be eliminated from (7) and (9). This gives

$$
\begin{aligned}
& \left\{\eta_{m} w u_{0}+\frac{2}{w R} \frac{V_{c}^{2}}{u_{0}}\right\}^{2} \\
& \quad+\left\{\left(K-M w^{2}+\frac{\Theta^{2}}{C_{p}}\right) u_{0}-\frac{\pi \Theta}{2 C_{p} w R} V_{c}\right\}^{2}=F_{0}^{2} .
\end{aligned}
$$

As the magnitude of displacement $u_{0}$ is related with the rectified voltage $V_{c}$ by (5), the above equation (10) can be further simplified to find $u_{0}$. The result is

$$
u_{0}=\frac{F_{0}}{\left\{\left(\eta_{m} w+\frac{2 w \Theta^{2} R}{\left(C_{p} w R+\frac{\pi}{2}\right)^{2}}\right)^{2}+\left(K-w^{2} M+\frac{w \Theta^{2} R}{C_{p} w R+\frac{\pi}{2}}\right)^{2}\right\}^{\frac{1}{2}}} .
$$

The average harvested power can also be obtained once $u_{0}$ is determined since

$$
P=\frac{V_{c}^{2}}{R}=\frac{w^{2} \Theta^{2} R}{\left(w C_{p} R+\frac{\pi}{2}\right)^{2}} u_{0}^{2} .
$$

To summarize, the normalized displacement $\bar{u}_{0}$, rectified voltage $\bar{V}_{c}$ and average harvested power $\bar{P}$ can be expressed by

$$
\begin{aligned}
\bar{u}= & \frac{u_{0}}{\frac{F_{0}}{K}} \\
= & \frac{1}{\left\{\left(2 \zeta_{m}+\frac{2 k_{e}^{2} r}{\left(r \Omega+\frac{\pi}{2}\right)^{2}}\right)^{2} \Omega^{2}+\left(1-\Omega^{2}+\frac{\Omega k_{e}^{2} r}{r \Omega+\frac{\pi}{2}}\right)^{2}\right\}^{\frac{1}{2}}}, \\
\bar{V}_{c}= & \frac{V_{c}}{\frac{F_{0}}{\Theta}}=\left(\frac{r \Omega}{r \Omega+\frac{\pi}{2}}\right) \\
& \times \frac{k_{e}^{2}}{\left\{\left(2 \zeta_{m}+\frac{2 k_{e}^{2} r}{\left(r \Omega+\frac{\pi}{2}\right)^{2}}\right)^{2} \Omega^{2}+\left(1-\Omega^{2}+\frac{\Omega k_{e}^{2} r}{r \Omega+\frac{\pi}{2}}\right)^{2}\right\}^{\frac{1}{2}}},
\end{aligned}
$$

$$
\begin{aligned}
\bar{P}= & \frac{P}{\frac{F_{0}^{2}}{w_{\mathrm{sc}} M}}=\frac{1}{\left(r \Omega+\frac{\pi}{2}\right)^{2}} \\
& \times \frac{k_{e}^{2} \Omega^{2} r}{\left\{\left(2 \zeta_{m}+\frac{2 k_{e}^{2} r}{\left(r \Omega+\frac{\pi}{2}\right)^{2}}\right)^{2} \Omega^{2}+\left(1-\Omega^{2}+\frac{\Omega k_{e}^{2} r}{r \Omega+\frac{\pi}{2}}\right)^{2}\right\}},
\end{aligned}
$$

where several non-dimensionless variables are introduced by

$$
\begin{array}{lll}
k_{e}^{2}=\frac{\Theta^{2}}{K C_{p}}, & \zeta_{m}=\frac{\eta_{m}}{2 \sqrt{K M}}, & w_{\mathrm{sc}}=\sqrt{\frac{K}{M}}, \\
\Omega=\frac{w}{w_{\mathrm{sc}}}, & r=C_{p} w_{\mathrm{sc}} R . &
\end{array}
$$

Above $k_{e}^{2}$ is the alternative electromechanical coupling coefficient $^{1}, \zeta_{m}$ the mechanical damping ratio, $w_{\mathrm{sc}}$ the natural frequency of the short circuit, $\Omega$ and $r$ the normalized frequency and electric resistance. Note that there are two resonances for the system since the piezoelectric structure exhibits both short circuit and open circuit stiffness. They are defined by

$$
\Omega_{\mathrm{sc}}=1, \quad \Omega_{\mathrm{oc}}=\sqrt{1+k_{e}^{2}},
$$

where $\Omega_{\mathrm{sc}}$ and $\Omega_{\mathrm{oc}}$ are the frequency ratios of the short circuit and the open circuit, respectively. Note that the shift in device natural frequency is pronounced if the coupling factor $k_{e}^{2}$ is large. Besides, (12), (13) and (14) are validated both numerically and experimentally, and are also compared well with other existing estimates in [34].

Since (14) is expressed in terms of non-dimensionless parameters, it can be used as the power normalization scheme to compare the performance and efficiency of the devices relatively. This is particularly useful in the design of a MEMSscale power generator since the testing, characterization and fabrication of micro-scale energy harvesters are not always available compared to the similar tasks in bulk power harvesters. Further, in most vibration-based power harvesting systems the vibration source is due to the periodic excitation of some base. This gives $F_{0}=M A$, where $A$ is the magnitude of acceleration of the exciting base. Therefore, the harvested average power per unit mass is described by

$$
\frac{P}{M}=\frac{A^{2}}{w_{\mathrm{sc}}} \bar{P}\left(r, \Omega, k_{e}^{2}, \zeta_{m}\right) .
$$

This shows that the harvested average power per unit mass depends on the input vibration characteristics (frequency ratio $\Omega$ and acceleration $A$ ), the normalized electric resistance $r$, the short circuit resonance $w_{\mathrm{sc}}$, the mechanical damping ratio $\zeta_{m}$ and the overall electromechanical coupling coefficient $k_{e}^{2}$ of the system. Thus, the scheme to optimize the power either by tuning the electric resistance, selecting suitable operation points, or adjusting the coupling coefficient by optimal structural design can be guided completely by (14).

\section{Conversion efficiency and electrically induced damping}

The efficiency of mechanical to electrical energy conversion is a fundamental parameter in order to compare energy harvesters of various sizes and with different vibration inputs. If the

\footnotetext{
1 The definition of $k_{e}^{2}$ here is slightly different from that used by [47].
} 
generator is excited by a harmonic forcing function as in (3), the energy conversion efficiency is commonly defined as the time-averaged power ratio by

eff $=\frac{W^{e}}{W^{i n}}, \quad W^{e}=f \frac{V_{c}^{2}}{R} \mathrm{~d} t, \quad W^{i n}=f F(t) \dot{u} \mathrm{~d} t$,

where $f \cdots \mathrm{d} t$ denotes the average over time [26, 33]. Above $W^{e}$ is the time-averaged power dissipated across the load resistor $R$ and $W^{i n}$ is the time-averaged power done by the external force. The balance of energy in (6) gives

$$
W^{i n}=W^{m}+W^{e}, \quad W^{m}=f \eta_{m} \dot{u}^{2} \mathrm{~d} t,
$$

where $W^{m}$ is the time-averaged power dissipated due to the structural damping. Further, the use of (7) provides an expression of efficiency of energy conversion under the steadystate operation. Indeed, it is given by

$$
\begin{aligned}
\text { eff } & =\frac{\frac{\pi}{w} \frac{V_{c}^{2}}{R}}{\frac{\pi}{2} \eta_{m} w u_{0}^{2}+\frac{\pi}{w} \frac{V_{c}^{2}}{R}} \\
& =\frac{2\left(\frac{V_{c}}{u_{0}}\right)^{2}}{\eta_{m} w^{2} R+2\left(\frac{V_{c}}{u_{0}}\right)^{2}} \\
& =\frac{2 \Theta^{2} R^{2} w^{2}}{\eta_{m} w^{2} R\left(w C_{p} R+\frac{\pi}{2}\right)^{2}+2 \Theta^{2} R^{2} w^{2}}
\end{aligned}
$$

due to (5). It can also be written in terms of the nondimensionless parameters defined in (15) by

$$
\text { eff }=\frac{r \frac{k_{e}^{2}}{\zeta_{m}}}{\left(r \Omega+\frac{\pi}{2}\right)^{2}+r \frac{k_{e}^{2}}{\zeta_{m}}} .
$$

It is clear from (19) that the conversion efficiency depends on the normalized resistance $r$, the applied frequency ratio $\Omega$ and, in particular, the relative magnitudes of the electromechanical coupling coefficient $k_{e}^{2}$ and the mechanical damping ratio $\zeta_{m}$. However, one has to be cautious when applying (19) to the study of conversion efficiency far below resonance since the dielectric loss term is not included in the present model. We refer to [29] which has studied the effect of dielectric loss on the efficiency of mechanical impact energy transformed into electric energy.

Williams and Yates [48] have proposed a model to study the conversion of the kinetic energy to electric power without specifying the mechanism by which the conversion process takes place. It is based on the idea that the conversion of energy from the oscillating mass to electricity is similar to a linear damper in the conventional mass-spring system. According to their model, the total damping ratio of the system can be decomposed as

$$
\zeta_{\text {tot }}=\zeta_{m}+\zeta_{e},
$$

where $\zeta_{e}$ is the electrically induced damping ratio due to the removal of mechanical energy from the vibrating system. Using the efficiency derived in (19), we can determine the induced damping $\zeta_{e}$ for an ac-dc piezoelectric power harvesting system. Indeed, the efficiency of the energy conversion can be re-defined by

$$
\text { eff }=\frac{\zeta_{e}}{\zeta_{m}+\zeta_{e}}
$$

if the effect of the electric system on the mechanical system is proportional to the velocity of the oscillating mass. Thus, from (19) and (20), the induced damping added to the system can be found to be

$$
\zeta_{e}=\frac{r k_{e}^{2}}{\left(r \Omega+\frac{\pi}{2}\right)^{2}} .
$$

Note that $\zeta_{e}$ is small at a small load resistance since only a slight fraction of energy is removed from the system. It is also small at a larger electric load since the circuit behaves like an open-circuit condition, preventing the generated charges from flowing out of the piezoelectric elements.

Another quantity known as the loss factor is commonly used for comparing the damping capacity of a vibrating system. It is defined as the ratio of the energy dissipated per radian and the total strain energy, and is related to the total damping ratio by

$$
\text { (loss factor })_{\text {tot }} \cong 2 \zeta_{\text {tot }}=2\left(\zeta_{m}+\zeta_{e}\right)
$$

for small values of damping [49]. Thus, the loss factor added to the system due to the energy dissipated across the load resistor is therefore

$$
(\text { loss factor })_{e} \cong 2 \zeta_{e}=\frac{2 r k_{e}^{2}}{\left(r \Omega+\frac{\pi}{2}\right)^{2}} .
$$

We now turn to the study of the optimal efficiency of energy conversion which is important in the design of an energy harvester. From (19), the normalized load $r^{\text {eff }}$ to maximize efficiency for fixed $\Omega, k_{e}^{2}, \zeta_{m}$ can be obtained according to

$$
\left.\frac{\partial}{\partial r} \operatorname{eff}\left(r, \Omega, k_{e}^{2}, \zeta_{m}\right)\right|_{\Omega, k_{e}^{2}, \zeta_{m}}=0
$$

This gives

$$
r^{\text {eff }}=\frac{\pi}{2 \Omega} \quad \text { and } \quad \text { eff }^{\max }=\frac{\frac{k_{e}^{2}}{\zeta_{m}}}{2 \pi \Omega+\frac{k_{e}^{2}}{\zeta_{m}}} .
$$

Besides, it can be shown from (21) that the electrically induced damping ratio evaluated at $r^{\text {eff }}$ has also achieved the maximum value; i.e.,

$$
\left.\zeta_{e}\right|_{r=r^{\mathrm{eff}}}=\zeta_{e}^{\max }=\frac{k_{e}^{2}}{2 \Omega \pi} .
$$

This in turn gives the maximum value of the electrically induced loss factor

$$
(\text { loss factor })_{e}^{\max } \cong 2 \zeta_{e}^{\max }=\frac{k_{e}^{2}}{\Omega \pi} .
$$

Lesieutre et al [37] have studied the induced electric damping associated with a piezoelectric energy harvesting system and derived an expression for the maximum loss factor. It is

$(\text { loss factor })_{e}^{\max }=\frac{k_{\text {sys }}^{2}}{\pi}, \quad$ (Lesieutre et al [37])

for small values of $k_{\text {sys }}^{2}$ which is defined by

$$
k_{\mathrm{sys}}^{2}=\frac{\Omega_{\mathrm{oc}}^{2}-\Omega_{\mathrm{sc}}^{2}}{\Omega_{\mathrm{oc}}^{2}}=\frac{k_{e}^{2}}{1+k_{e}^{2}} .
$$


Obviously, (25) and (26) are almost identical provided that the coupling coefficient $k_{e}^{2}$ is small and the applied frequency ratio $\Omega \approx 1$. Note that Lesieutre et al [37] have derived an expression of the maximum loss factor by assuming that it occurs at the condition of the optimal power transfer. Their argument is in generally true for most common cases except the situation where the ratio $\frac{k_{e}^{2}}{\zeta_{m}}$ is large. We will discuss it in the next section.

\section{Discussion}

The shift in device natural frequency from $\Omega_{\mathrm{sc}}$ to $\Omega_{\mathrm{oc}}$ is pronounced if the electromechanical coupling coefficient $k_{e}^{2}$ is large, as seen from (16). It occurs often in devices whose the piezoelectric element's contribution to the overall structural stiffness is significant [25]. In addition, this frequency shift could be large at the piezoelectric micro power generator operated utilizing the $\{3-3\}$ mode since the piezoelectric effect is further enhanced in the longitudinal mode [5, 25]. The consequence of pronounced shift in natural frequency from $\Omega_{\mathrm{sc}}$ to $\Omega_{\mathrm{oc}}$ leads to completely distinct optimization schemes for maximum power extraction. Shu and Lien [34] have studied the ac-dc power output for a rectified piezoelectricbased harvesting device. They have shown that the average harvested power has two identical peaks evaluated at two different electric loads at the respective operating points provided that $\frac{k_{e}^{2}}{\zeta_{m}} \gg 1$ while it has only one peak otherwise. Thus, the study of relation among the conversion efficiency, electrically induced damping and power transfer has to be classified according to the relative magnitudes of the coupling coefficient and the mechanical damping ratio.

\subsection{Weak electromechanical coupling}

In the following, we take $k_{e}^{2}=0.16$ and $\zeta_{m}=0.03$ which are typical parameters for piezoelectric power generators designed as the cantilever-beam type and operated in the $\{3-1\}$ mode.

The conversion efficiency, normalized displacement and harvested power against the frequency ratio are plotted in figure $4(a)-(c)$ for various normalized resistances. In addition, they are also plotted versus normalized resistance, with varying frequency ratios in figures $4(d)-(f)$. Consider figures $4(a)$ and $(d)$ first. The conversion efficiency is small and around $18 \%$ for very small load at $r=0.1$, and is increasing as the resistance becomes large. According to (23), it achieves its maximum value

$$
\operatorname{eff}^{\max } \approx \frac{\frac{k_{e}^{2}}{\zeta_{m}}}{2 \pi+\frac{k_{e}^{2}}{\zeta_{m}}}=46 \%
$$

at $r^{\text {eff }}=\frac{\pi}{2 \Omega}=1.57$ for $\Omega \approx 1$. The conversion efficiency then decreases as the load exceeds $r^{\text {eff }}$. Besides, the overlapping of curves in figure $4(d)$ shows that the efficiency of energy conversion is not sensitive with respect to the frequency ratio in the case of the weak electromechanical coupling.

We next consider figures $4(b)$ and $(e)$. Each curve of displacement has a peak around the resonance whose value depends on the resistance. From (21) the electrically induced damping $\zeta_{e}$ is small at both small and large electric loads while it attains its maximum value at $r^{\text {eff }}$, which in turn also gives the maximum conversion efficiency of the generator. As a result, the peak of displacement descends until to the point which corresponds to the maximum induced damping as well as efficiency. When $r$ moves past $r^{\text {eff }}$, the induced damping decreases, resulting in the elevation of the peak of displacement. Figure 4(e) illustrates the dependence of displacement on resistance at the different operating points. The displacement becomes monotonically decreasing if the device is excited in the vicinity of its short circuit resonance while it becomes monotonically increasing at the open circuit resonance. If the frequency is operated around the middle point of $\Omega_{\mathrm{sc}}$ and $\Omega_{\mathrm{oc}}$, the curve has a local minimum at the turning point close to $r^{\text {eff }}$ where the induced damping is maximized.

Note that our theoretical prediction on the behaviour of displacement against frequency ratio for various electric loads, as illustrated in figure $4(b)$, qualitatively agrees well with the experimental results observed by Lesieutre et al (see figure 6 in [37]).

Each curve of harvested power has a maximum around the resonance whose value depends on the resistance, as demonstrated in figure 4(c). In contrast with displacement, the peak of power and the electrically induced damping $\zeta_{e}$ simultaneously ascend as the electric load increases. It attains the maximum value at $r^{\text {eff }}$ corresponding to the local minimum of displacement as well as the maximum of efficiency. After that, the peak of power descends when the load exceeds $r^{\text {eff }}$. Finally, the optimal load to maximize the harvested power is illustrated in figure $4(f)$ with varying frequency ratios. The peaks of power are slightly higher for frequencies operated around $\Omega_{\mathrm{sc}}$ than those operated around $\Omega_{\mathrm{oc}}$. However, unlike the conversion efficiency in figure $4(d)$ which is insensitive to the frequency ratio, a small derivation from the optimal load results in a more significant drop in power operated around $\Omega_{\mathrm{sc}}$ than that operated around $\Omega_{\mathrm{oc}}$.

\subsection{Strong electromechanical coupling}

We use $k_{e}^{2}=1$ and $\zeta_{m}=0.025$ here to demonstrate the effect of strong coupling on the relation among the efficiency, damping and the power transfer.

The conversion efficiency, normalized displacement and power versus frequency ratio are plotted, with varying load resistances in figures $5(a)-(c)$. Besides, they are also plotted against normalized resistance in figures $5(d)-(f)$ for a variety of frequency ratios. Unlike the case of weak coupling, the average harvested power has two identical optimal peaks evaluated at different resistances and frequencies; i.e.,

$$
\max _{r, \Omega} \bar{P}(r, \Omega)=\bar{P}\left(r_{1}^{\mathrm{opt}}, \Omega_{1}^{\mathrm{opt}}\right)=\bar{P}\left(r_{2}^{\mathrm{opt}}, \Omega_{2}^{\mathrm{opt}}\right),
$$

where

$$
\begin{aligned}
& \left(r_{1}^{\text {opt }}, \Omega_{1}^{\text {opt }}\right)=(0.07,1.02), \\
& \left(r_{2}^{\text {opt }}, \Omega_{2}^{\text {opt }}\right)=(18.27,1.39) .
\end{aligned}
$$

Obviously, $\Omega_{1}^{\mathrm{opt}} \approx \Omega_{\mathrm{sc}}=1, \Omega_{2}^{\mathrm{opt}} \approx \Omega_{\mathrm{oc}}=1.41$ as illustrated in figure $5(c)$, and $r_{1}^{\text {opt }} \ll 1, r_{2}^{\text {opt }} \gg 1$ as demonstrated in figure $5(f)$. In contrast with figure $4(c)$, the peak of harvested power decreases while the electrically induced damping $\zeta_{e}$ increases as the load exceeds $r_{1}^{\text {opt }}$. The envelope of these peaks 


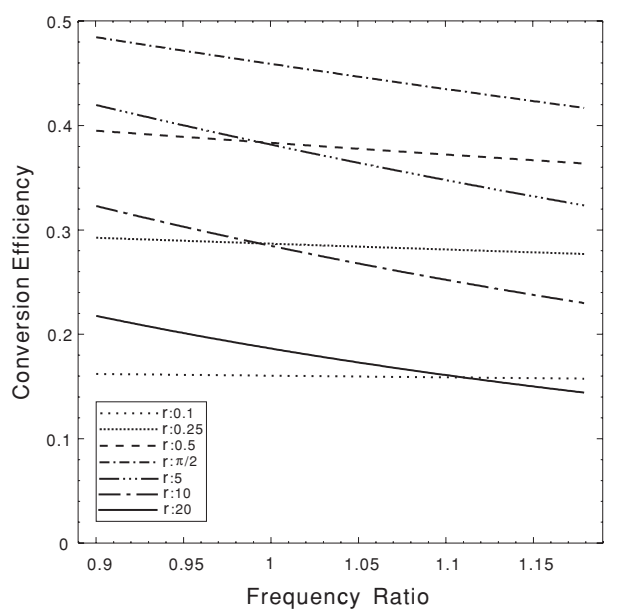

(a)

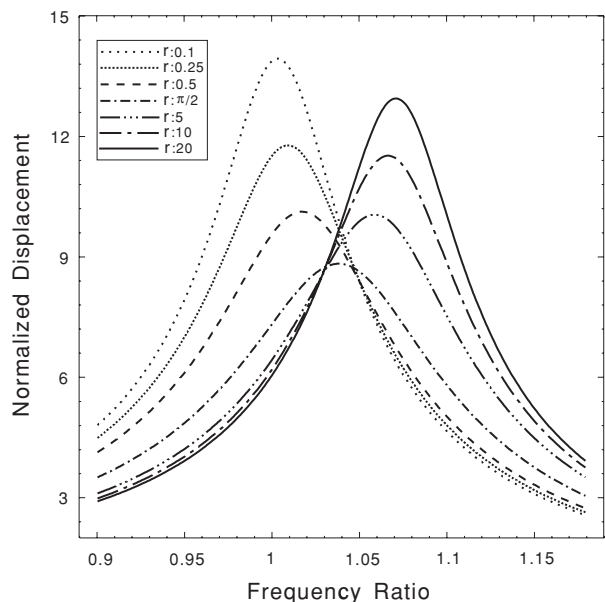

(b)

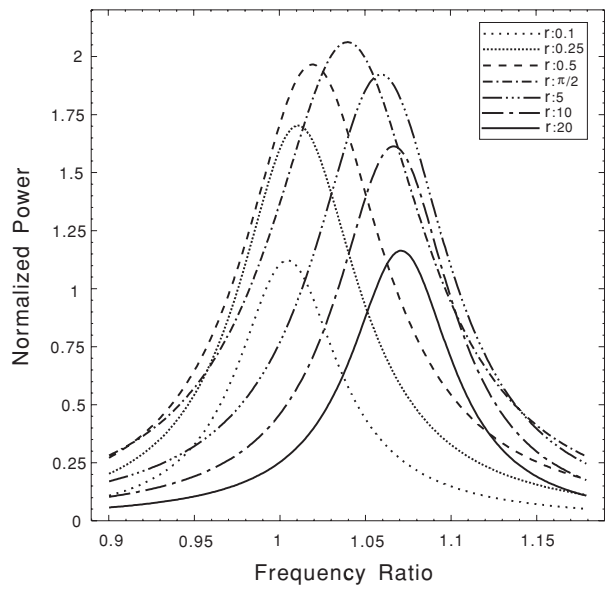

(c)

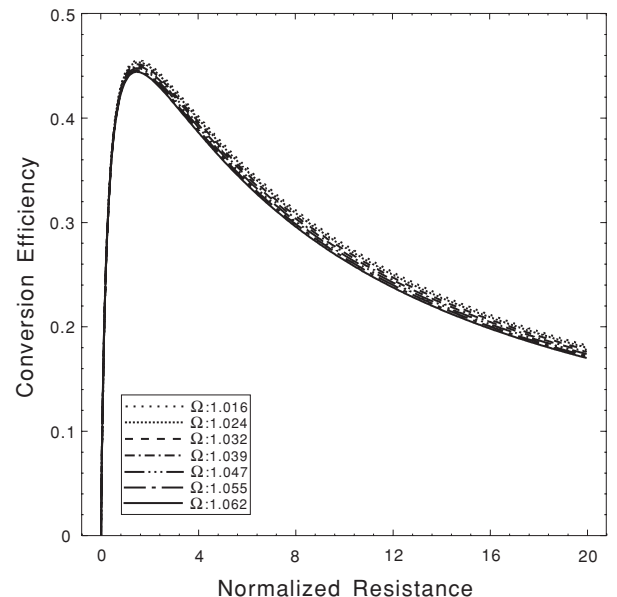

(d)

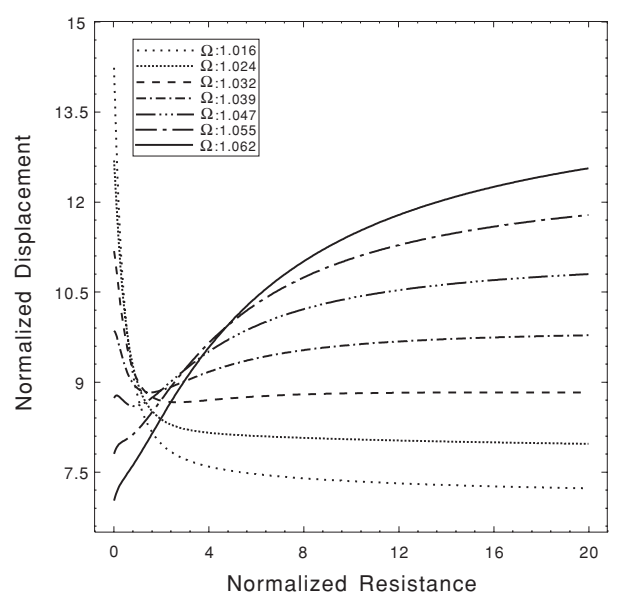

(e)

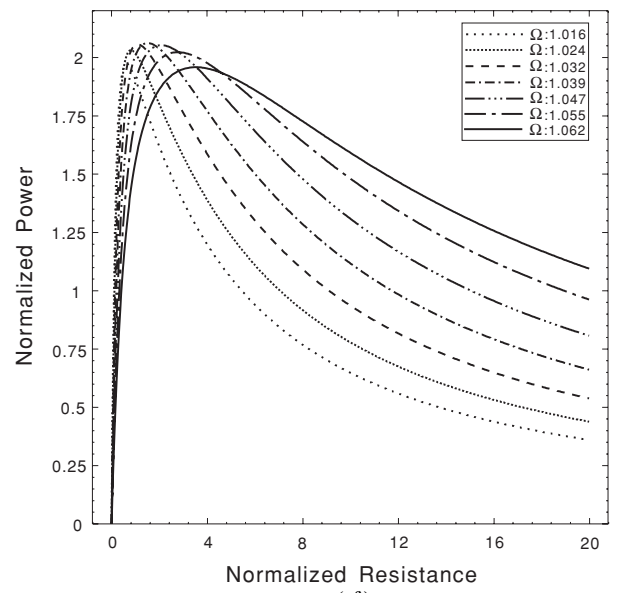

$(f)$

Figure 4. Weak electromechanical coupling demonstrated using $k_{e}^{2}=0.16$ and $\zeta_{m}=0.03:(a),(b)$ and $(c)$ are the conversion efficiency, displacement and power against frequency ratio for various resistances, while $(d),(e)$ and $(f)$ are those against resistance with varying frequency ratios.

has a local minimum which is closely related to the maximum induced damping $\zeta_{e}$. When the load further increases, the peak of power then rises to the second maximum, closely corresponding to $\Omega_{\mathrm{oc}}$ as described by (29). Figure 5( $f$ ) shows the dependence of the harvested power versus the normalized resistance for a variety of frequency ratios. Switching between these two peaks, corresponding to $r_{1}^{\text {opt }}$ and $r_{2}^{\text {opt }}$, is attained by varying the applied frequency from $\Omega_{\mathrm{sc}}$ to $\Omega_{\mathrm{oc}}$. It indicates the importance of selecting the correct operating point in the case of strong coupling.

The dependence of conversion efficiency on the frequency as well as on the load resistance in the strong coupling case is qualitatively similar to the case of weak coupling. But the magnitude of conversion efficiency is much higher here 


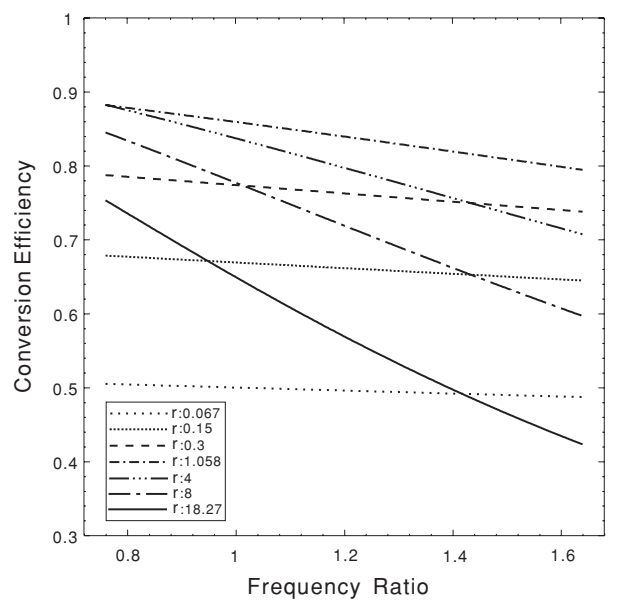

(a)

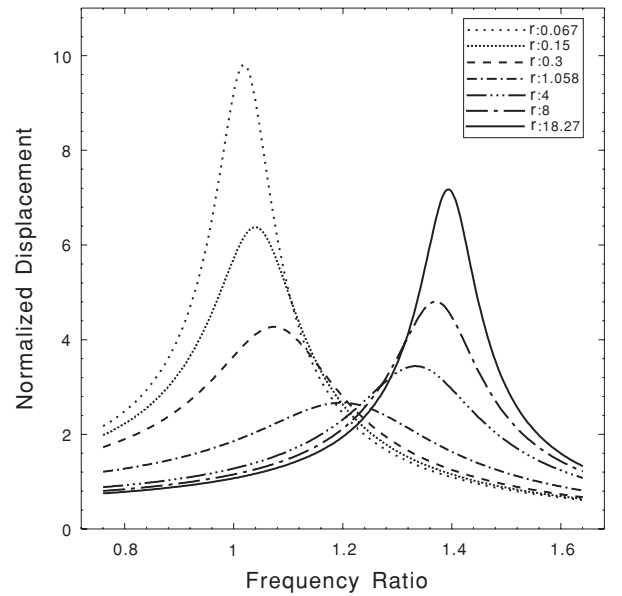

(b)

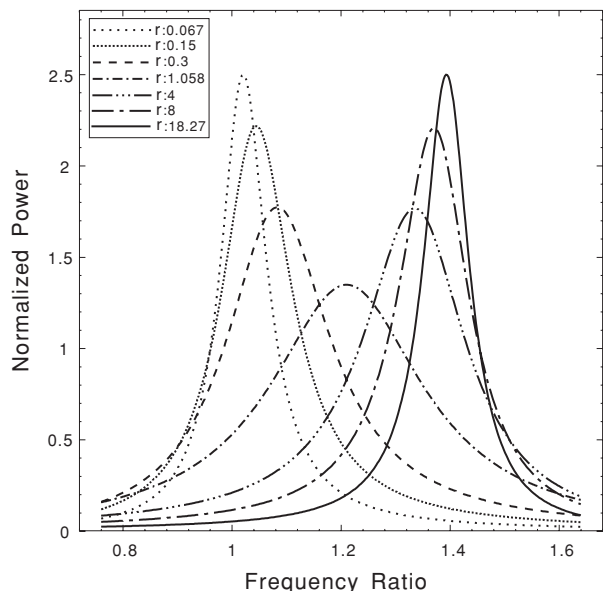

(c)

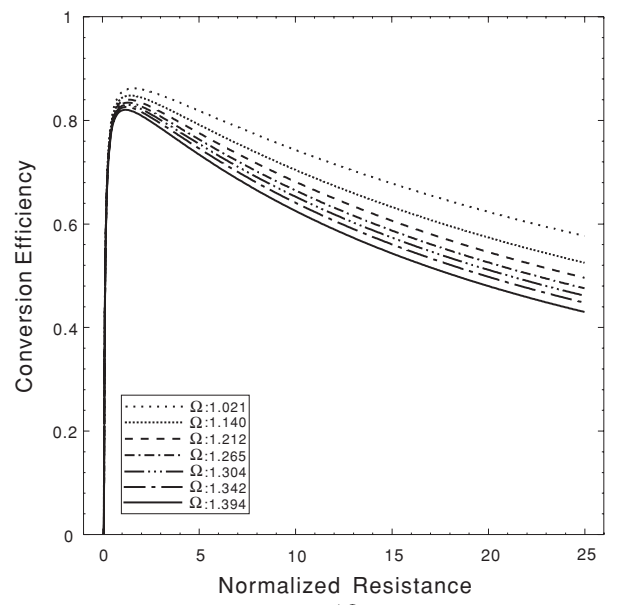

(d)

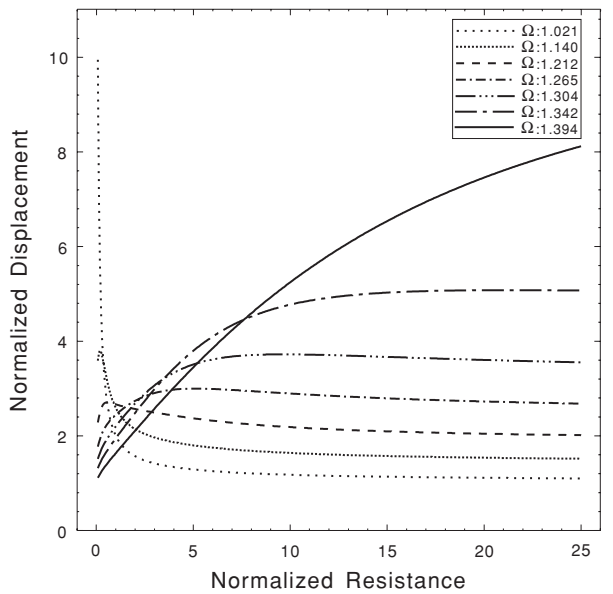

(e)

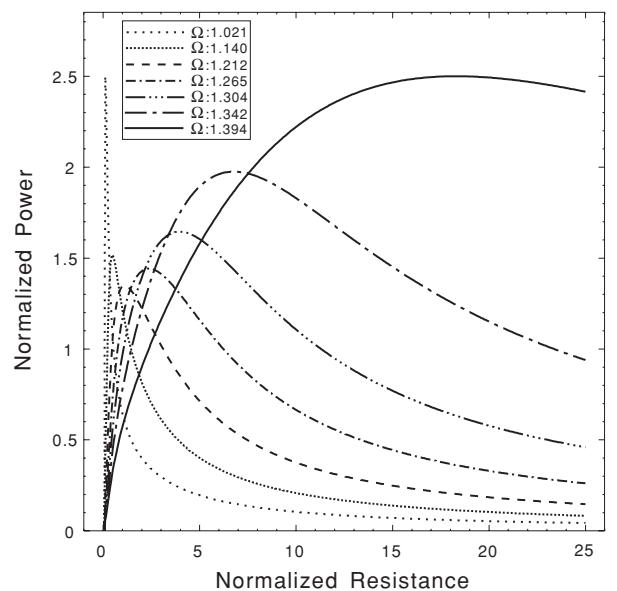

(f)

Figure 5. Strong electromechanical coupling demonstrated using $k_{e}^{2}=1$ and $\zeta_{m}=0.025:(a),(b)$ and $(c)$ are the conversion efficiency, displacement and power against frequency ratio for various resistances, while $(d),(e)$ and $(f)$ are those against resistance with varying frequency ratios.

because of the relatively large ratio $\frac{k_{e}^{2}}{\zeta_{m}}$. Our current example points out eff ${ }^{\max } \approx 90 \%$ which is much larger than that $(46 \%$ in (27)) in the weak coupling case. Besides, the condition to maximize the conversion efficiency does not lead to the maximum power transfer in the strong electromechanical coupling; instead, it is closely related to the local minimum of the power envelope. The conversion efficiency takes on the smaller value around $50 \%$ at the optimal power generation, as shown in figures $5(d)$ and $(f)$.

Finally, we consider figures $5(b)$ and $(e)$. The effect of the induced electric damping is much pronounced in the strong coupling case than that in the case of weak coupling since, from $(21), \zeta_{e}$ is proportional to $k_{e}^{2}$. As a result, at the maximum induced damping, the envelope of the peaks of displacement 
has a local minimum whose value is much smaller than that of the first peak. Figure 5(b) demonstrates that the peak of displacement decreases approximately from 10 to 2 at the maximum induced damping, while the weak coupling case shows a moderate descent in figure $4(b)$. Unlike the power, the second maximum of these peaks due to the reduction of $\zeta_{e}$ at large $r$ is smaller than the first one since the system has more damping at the open-circuit condition. Finally, in contrast with the results obtained in the weak coupling case, the two pronounced peaks of displacement evaluated around $\Omega_{\mathrm{sc}}$ and $\Omega_{\mathrm{oc}}$ correspond to the maximum extraction of power rather than the local minimum point of the envelope of displacement by comparing figures $5(b)$ and $(c)$ with figures $4(b)$ and $(c)$.

\section{Conclusion}

This paper establishes the relation among the energy conversion efficiency, electrically induced damping and power transfer for a rectified piezoelectric power harvester. An analytical model is proposed, and an exact formula for the conversion efficiency is derived under the steady-state operation. It is shown that the efficiency depends on the normalized resistance $r$, the frequency ratio $\Omega$ and, in particular, the relative magnitudes of the electromechanical coupling coefficient $k_{e}^{2}$ and the mechanical damping ratio $\zeta_{m}$. In general, high energy conversion efficiency can be achieved with large $\frac{k_{e}^{2}}{\zeta_{m}}$; the improvement of the coupling coefficient $k_{e}^{2}$ for micromachined piezoelectric membrane harvesters has been recently investigated by Cho et al $[35,36]$.

The induced damping added to the system due to the removal of mechanical energy from the vibrating structure is obtained based on the derived formula of conversion efficiency. It is shown that the maximum conversion efficiency corresponds to the maximum induced electric damping as well as the optimal power transfer in the case of weak electromechanical coupling. This result is consistent with that observed by Lesieutre et al [37].

However, unlike [37], a new finding shows that the optimal electric load maximizing the conversion efficiency and induced electric damping is very different from that maximizing the harvested power in strongly coupled electromechanical systems. This gives completely distinct optimization schemes, since the harvested power has two identical peaks evaluated at two different electric loads at the respective operating points, provided that $\frac{k_{e}^{2}}{\zeta_{m}} \gg 1$, while it has only one peak otherwise.

\section{Acknowledgments}

We are glad to acknowledge the Ministry of Economic Affairs for support under grant no 94-EC-17-A-05-S1-017 (WHAM$\mathrm{BioS})$.

\section{References}

[1] Chandrakasan A, Amirtharajah R, Goodman J and Rabiner W 1998 Trends in low power digital signal processing Int. Symp. Circuits Syst. 4 604-7

[2] Roundy S, Steingart D, Frechette L, Wright P and Rabaey J 2004 Power sources for wireless sensor networks Lecture Notes Comput. Sci. 2920 1-17
[3] Roundy S, Leland E S, Baker J, Carleton E, Reilly E, Lai E, Otis B, Rabaey J M, Wright P K and Sundararajan V 2005 Improving power output for vibration-based energy scavengers IEEE Pervasive Comput. 4 28-36

[4] Sodano H A, Inman D J and Park G 2004 A review of power harvesting from vibration using piezoelectric materials Shock Vib. Dig. 36 197-205

[5] Jeon Y B, Sood R, Jeong J H and Kim S G 2005 MEMS power generator with transverse mode thin film PZT Sensors Actuators A 122 16-22

[6] Poulin G, Sarraute E and Costa F 2004 Generation of electric energy for portable devices: comparative study of an electromagnetic and a piezoelectric system Sensors Actuators A 116 461-71

[7] Roundy S, Wright P K and Rabaey J 2003 A study of low level vibrations as a power source for wireless sensor nodes Comput. Commun. 26 1131-44

[8] Sodano H A, Inman D J and Park G 2005 Generation and storage of electricity from power harvesting devices J. Intell. Mater. Syst. Struct. 16 67-75

[9] Elvin N G, Elvin A A and Spector M 2001 A self-powered mechanical strain energy sensor Smart Mater. Struct. 10 293-9

[10] Elvin N G, Lajnef N and Elvin A A 2006 Feasibility of structural monitoring with vibration powered sensors Smart Mater. Struct. 15 977-86

[11] Ng T G and Liao W H 2005 Sensitivity analysis and energy harvesting for a self-powered piezoelectric sensor J. Intell. Mater. Syst. Struct. 16 785-97

[12] Roundy S and Wright P K 2004 A piezoelectric vibration based generator for wireless electronics Smart Mater. Struct. $131131-42$

[13] Sodano H A, Inman D J and Park G 2005 Comparison of piezoelectric energy harvesting devices for recharging batteries J. Intell. Mater. Syst. Struct. 16 799-807

[14] Sodano H A, Lloyd J and Inman D J 2006 An experimental comparison between several active composite actuators for power generation Smart Mater. Struct. 15 1211-6

[15] White N M, Glynne-Jones P and Beeby S P 2001 A novel thick-film piezoelectric micro-generator Smart Mater. Struct. 10 850-2

[16] Ericka M, Vasic D, Costa F, Poulin G and Tliba S 2005 Energy harvesting from vibration using a piezoelectric membrane $J$. Physique IV 128 187-93

[17] Kim S, Clark W W and Wang Q M 2005 Piezoelectric energy harvesting with a clamped circular plate: analysis J. Intell. Mater. Syst. Struct. 16 847-54

[18] Kim S, Clark W W and Wang Q M 2005 Piezoelectric energy harvesting with a clamped circular plate: experimental study J. Intell. Mater. Syst. Struct. 16 855-63

[19] Whalen S, Thompson M, Bahr D, Richards C and Richards R 2003 Design, fabrication and testing of the $\mathrm{P}^{3}$ micro heat engine Sensors Actuators A $104290-8$

[20] Allen J J and Smits A J 2001 Energy harvesting EEL J. Fluids Struct. 15 629-40

[21] Taylor G W, Burns J R, Kammann S M, Powers W B and Welsh T R 2001 The energy harvesting Eel: a small subsurface ocean/river power generator IEEE J. Ocean. Eng. 26 539-47

[22] Kim H W, Batra A, Priya S, Uchino K, Markley D, Newnham R E and Hofmann H F 2004 Energy harvesting using a piezoelectric 'Cymbal' transducer in dynamic environment Japan. J. Appl. Phys. 43 6178-83

[23] Kim H W, Priya S, Uchino K and Newnham R E 2005 Piezoelectric energy harvesting under high pre-stressed cyclic vibrations J. Electroceram. 15 27-34

[24] Priya S, Chen C T, Fye D and Zahnd J 2005 Piezoelectric windmill: a novel solution to remote sensing Japan. J. Appl. Phys. 44 L104-7

[25] duToit N E, Wardle B L and Kim S G 2005 Design considerations for MEMS-scale piezoelectric mechanical vibration energy harvesters Integr. Ferroelectr. 71 121-60 
[26] F Lu, Lee H P and Lim S P 2004 Modeling and analysis of micro piezoelectric power generators for micro-electromechanical-systems applications Smart Mater. Struct. 13 57-63

[27] Ramsay M J and Clark W W 2001 Piezoelectric energy harvesting for bio MEMS applications Proc. SPIE 4332 429-38

[28] Horowitz S B, Sheplak M, Cattafesta L N III and Nishida T 2006 A MEMS acoustic energy harvester J. Micromech. Microeng. 16 S174-81

[29] Umeda M, Nakamura K and Ueha S 1996 Analysis of the transformation of mechanical impact energy to electric energy using piezoelectric vibrator Japan. J. Appl. Phys. 35 3267-73

[30] Umeda M, Nakamura K and Ueha S 1997 Energy storage characteristics of a piezo-generator using impact induced vibration Japan. J. Appl. Phys. 36 3146-51

[31] Goldfarb M and Jones L D 1999 On the efficiency of electric power generation with piezoelectric ceramic Trans. ASME J. Dynamic Syst. Meas. Control 121 566-71

[32] Roundy S 2005 On the effectiveness of vibration-based energy harvesting J. Intell. Mater. Syst. Struct. 16 809-23

[33] Richards C D, Anderson M J, Bahr D F and Richards R F 2004 Efficiency of energy conversion for devices containing a piezoelectric component J. Micromech. Microeng. $14717-21$

[34] Shu Y C and Lien I C 2006 Analysis of power output for piezoelectric energy harvesting systems Smart Mater. Struct. 15 1499-512

[35] Cho J, Anderson M, Richards R, Bahr D and Richards C 2005 Optimization of electromechanical coupling for a thin-film PZT membrane: I. Modeling J. Micromech. Microeng. 15 1797-803

[36] Cho J, Anderson M, Richards R, Bahr D and Richards C 2005 Optimization of electromechanical coupling for a thin-film PZT membrane: II. Experiment J. Micromech. Microeng. 15 1804-9

[37] Lesieutre G A, Ottman G K and Hofmann H F 2004 Damping as a result of piezoelectric energy harvesting J. Sound Vib. 269 991-1001
[38] Sodano H A, Park G and Inman D J 2004 Estimation of electric charge output for piezoelectric energy harvesting J. Strain 40 49-58

[39] Ottman G K, Hofmann H F, Bhatt A C and Lesieutre G A 2002 Adaptive piezoelectric energy harvesting circuit for wireless remote power supply IEEE Trans. Power Electron. 17 669-76

[40] Ottman G K, Hofmann H F and Lesieutre G A 2003 Optimized piezoelectric energy harvesting circuit using step-down converter in discontinuous conduction mode IEEE Trans. Power Electron. 18 696-703

[41] Badel A, Benayad A, Lefeuvre E, Lebrun L, Richard C and Guyomar D 2006 Single crystals and nonlinear process for outstanding vibration-powered electrical generators IEEE Trans. Ultrason. Ferroelectr. Freq. Control 53 673-84

[42] Badel A, Guyomar D, Lefeuvre E and Richard C 2005 Efficiency enhancement of a piezoelectric energy harvesting device in pulsed operation by synchronous charge inversion J. Intell. Mater. Syst. Struct. 16 889-901

[43] Guyomar D, Badel A and Lefeuvre E 2005 Toward energy harvesting using active materials and conversion improvement by nonlinear processing IEEE Trans. Ultrason. Ferroelectr. Freq. Control 52 584-95

[44] Lefeuvre E, Badel A, Richard C and Guyomar D 2005 Piezoelectric energy harvesting device optimization by synchronous electric charge extraction J. Intell. Mater. Syst. Struct. 16 865-76

[45] Hagood N W, Chung W H and Flotow A V 1990 Modelling of piezoelectric actuator dynamics for active structural control J. Intell. Mater. Syst. Struct. $1327-54$

[46] Wang Q M and Cross L E 1999 Constitutive equations of symmetrical triple layer piezoelectric benders IEEE Trans. Ultrason. Ferroelectr. Freq. Control 46 1343-51

[47] Lesieutre G A and Davis C L 1997 Can a coupling coefficient of a piezoelectric device be higher than those of its active material? J. Intell. Mater. Syst. Struct. 8 859-67

[48] Williams C B and Yates R B 1996 Analysis of a micro-electric generator for microsystems Sensors Actuators A 52 8-11

[49] Rao S S 1990 Mechanical Vibrations (Reading, MA: Addison-Wesley) 